\title{
Progress in Clinical Neurosciences A Systematic Review of the Use of Triptans in Acute Migraine
}

\author{
Marek J. Gawel, Irene Worthington, Anne Maggisano
}

\begin{abstract}
Objective: A systematic review of the literature was undertaken, to consolidate evidence concerning the efficacy and safety of triptans currently available in Canada (sumatriptan, rizatriptan, naratriptan, zolmitriptan), and to provide guidelines for selection of a triptan. Methods: Data from published, randomized, placebo-controlled trials were pooled and a combined number needed to treat (NNT) and number needed to harm (NNH) was generated for each triptan. Direct comparative trials of triptans were also examined. Results: The lowest NNTfor headache response/pain-free at one/two hours is observed with subcutaneous sumatriptan. Among the oral formulations, the lowest NNT is observed with rizatriptan and the highest NNT with naratriptan. The lowest NNH is observed with subcutaneous sumatriptan. Conclusions: Triptans are relatively safe and effective medications for acute migraine attacks. However, differences among them are relatively small. Considerations in selecting a triptan include individual patient response/tolerance, characteristics of the attacks, relief of associated symptoms, consistency of response, headache recurrence, delivery systems and patient preference.
\end{abstract}

RÉSUMÉ: Revue systématique de l'utilisation des triptans dans la migraine aiguë et lignes directrices. Objectif: Nous avons procédé à une revue systématique de la littérature pour consolider les données pertinentes à l'efficacité et à la sécurité des triptans disponibles au Canada (sumatriptan, rizatriptan, naratriptan, zolmitriptan), et pour élaborer des lignes directrices quant à la sélection d'un triptan. Méthodes: Les données des études randomisées ayant un groupe placebo, qui ont été publiées, ont été regroupées et un NNT et NNH combiné a été déterminé pour chaque triptan (NNT: number needed to treat - nombre d'individus à traiter pour prévenir un incident; NNH: number needed to harm - nombre d'individus à traiter pour qu'en moyenne un effet indésirable survienne). Les essais comparatifs de triptans ont également été examinés. Résultats: Le NNTle plus bas, pour la réponse définie comme l'absence de céphalée après une à deux heures, est observé avec l'administration sous-cutanée de sumatriptan. En ce qui concerne les préparations orales, le NNT le plus bas a été observé avec le rizatriptan et le plus élevé avec le naratriptan. Le NNH le plus bas a été observé avec le sumatriptan sous-cutané. Conclusions: Les triptans sont des médicaments relativement sûrs et efficaces pour traiter les accès aigus de migraine. Cependant, les différences entre eux sont relativement faibles. Les aspects à considérer dans le choix d'un triptan sont: la réponse et la tolérance du patient, les caractéristiques des accès, le soulagement des symptômes associés, la constance de la réponse, la récurrence de la céphalée, le mode d'administration et la préférence du patient.

Can. J. Neurol. Sci. 2001; 28: 30-41

In the mid-1980s, a new approach to the treatment of migraine was researched, in the form of the first relatively selective $5-\mathrm{HT}_{1 \mathrm{~B} / 1 \mathrm{D}}$ receptor agonist, sumatriptan. Sumatriptan was designed as an analogue of the serotonin molecule. The first formulation of sumatriptan, introduced in 1991, was a subcutaneous injection; this was followed by the oral and finally, the intranasal preparations. It became evident that sumatriptan was an effective treatment of acute migraine, both with and without aura. ${ }^{1,2}$ The introduction of sumatriptan revolutionized the treatment of acute migraine and led to an improved quality of life for patients who suffer from this debilitating neurological disorder.
Following the release of sumatriptan, other molecules of the same class were developed. These became known as the 'triptans'. In Canada, naratriptan, rizatriptan and zolmitriptan are

From the Division of Neurology (MJG), and Metro Toronto Hospitals Drug Information Service (MetroDIS) (IW, AM), Sunnybrook \& Women's College Health Sciences Centre, Toronto, ON Canada

Received July 14, 2000. ACCePtedin FinAlform OCtOBer 26, 2000. Reprint requests to: Marek J. Gawel, Division of Neurology, Sunnybrook \& Women's College Health Sciences Centre, 2075 Bayview Avenue, Toronto, ON Canada M4N 3M5 
currently available. Eletriptan, frovatriptan and almotriptan are not currently marketed in Canada.

Since sumatriptan was the first available triptan, it has the most extensive data supporting its efficacy, safety and tolerability. A systematic review of the efficacy and adverse effects of subcutaneous, oral and intranasal sumatriptan has been recently published. ${ }^{3}$ Although the newer triptans have less extensive data, they possess features such as improved bioavailability, increased lipophilicity and central nervous system penetration and, in the case of naratriptan, a longer halflife.

In 1997 and 1998, the Canadian Headache Society published guidelines for the diagnosis and management of migraine (pharmacologic) and for the nonpharmacologic management of migraine in adults. ${ }^{4,5}$ At the time of publication of the guidelines, sumatriptan was the only available triptan. This review will give information on triptans currently available in Canada.

\section{Purpose of The ReVieW}

This review is an attempt to consolidate evidence concerning the efficacy and tolerability of triptans currently available in Canada (i.e., sumatriptan, naratriptan, zolmitriptan, rizatriptan) for the acute or symptomatic treatment of migraine and to provide some guidelines for the selection of a triptan. Randomized, placebo-controlled studies and direct comparative studies (published in full) were primarily selected for review (see Methodology). These studies are available in the public domain. Data are often presented in the form of abstracts or posters at medical meetings; review of such data has not been included, since it is difficult to evaluate.

\section{Methodology}

A search of the MEDLINE database from 1980 - March 2000 was conducted. Randomized, placebo- or comparator-controlled trials, published in full, were primarily selected for the review. Other references quoted include: guidelines, systematic reviews, open label studies, retrospective studies, population-based studies, pharmacokinetic/drug interaction studies, manufacturers product monographs and letters to the editor. MeSH headings/textwords used included: migraine, sumatriptan, naratriptan, zolmitriptan, rizatriptan. Data from randomized, placebo-controlled trials were pooled for each individual triptan (separately for each strength and dosage form, excluding naratriptan $1 \mathrm{mg}$ oral and rizatriptan $5 \mathrm{mg}$ oral). Statistical assessment of homogeneity of trials was not performed. Statistical variables were calculated using EXCEL version 5.0:

1. Therapeutic gain: the percentage response to the active drug minus the percentage response to placebo at the same time point in the study.

2. Number needed to treat (NNT): the reciprocal of therapeutic gain. The number of people needed to treat with active drug before a positive response is observed (over and above the response provided by placebo).

3. Therapeutic penalty: the percentage of subjects experiencing an adverse event with the drug minus the percentage experiencing an adverse event with placebo (all recorded adverse events).

4. Number needed to harm (NNH): the reciprocal of therapeutic penalty. The number of subjects needed to treat with active drug before a negative event is observed.

5. 95\% confidence intervals (CI): calculated using the following formulae:

Confidence interval of the proportion ( $p$ ) of responders:

$95 \%$ CI $(\mathrm{p})=\mathrm{p} \pm 1.96 \sqrt{\mathrm{p}(1-\mathrm{p}) / \mathrm{n}}$

Confidence interval of the therapeutic gain or therapeutic penalty: placebo response (p) minus active response (a)

$95 \%$ CI $(\mathrm{p}-\mathrm{a})=(\mathrm{p}-\mathrm{a}) \pm 1.96 \sqrt{\mathrm{p}(1-\mathrm{p}) / \mathrm{n}+\mathrm{a}(1-\mathrm{a}) / \mathrm{m}}$

Confidence interval of the NNT (or NNH): the reciprocal of the CI for the therapeutic gain (or penalty).

Randomized controlled trials excluded from the analysis included those utilizing doses not currently available or recommended, nonstandard endpoint measurements (e.g., headache relief at four hours), special populations (e.g., menstrual migraine) and those that were not placebo-controlled.

\section{Efficacy}

Problems in the assessment of efficacy arose during the development of the triptans. A simplistic verbal response scale, with patients reporting severe, moderate, mild or no pain, superceded traditional methods of assessing pain relief based on visual analog scales. A 'headache response' is classified as a reduction in headache pain from severe or moderate to mild or none; therefore, patients who have a reduction of pain from moderate to mild are classified as 'responders'. A much more robust method of assessing efficacy is based on the concept of 'pain-free'. Studies also vary in the predetermined time point for assessing efficacy; two hours is the usual endpoint. Efficacy at one hour can now be assessed with the introduction of drugs that are more rapidly absorbed. However, for naratriptan, which is slowly absorbed, four-hour data has been primarily reported in clinical trials.

The endpoints for efficacy include:

1. Headache response at one or two hours: the percentage of patients whose headache severity is reduced from 'severe or moderate' at baseline to 'mild or none', one or two hours after taking a dose.

2. Pain-free at one or two hours: the percentage of patients whose headache severity is reduced from 'severe or moderate'at baseline to 'none', one or two hours after taking a dose.

3. Clinical disability score of 0/1 at two hours: the percentage of patients who rate their daily activities as 'mildly impaired'or 'normal', two hours after taking a dose.

4. Recurrence: the percentage of patients who, having obtained a response or freedom from headache pain within two hours of taking a dose, develop a worsening (moderate/severe headache pain) within 24 hours of the initial dose.

\section{Adverse events}

Reporting of adverse events has also been a problem. Most studies quote the incidence of adverse events occurring above a certain predetermined percentage, which varies from study to study (e.g., greater than 3\%, 5\%, etc.). The categorization of adverse events has also been difficult to unify. Different studies have quoted different types of adverse events. In some studies, adverse events have been captured from patient diaries, while others have been collected retrospectively. 
The endpoints for safety/tolerability include:

Overall incidence of adverse events: the percentage of patients who, having taken the drug, experience an unexpected or undesired event (includes all adverse events, not just drug-related).

\section{Pharmacology of The TRIPTANS}

The triptans are serotonin derivatives displaying highly selective and potent agonist activity at the vascular $5-\mathrm{HT}_{1 \mathrm{~B}}$ receptor and the neuronal 5- $\mathrm{HT}_{1 \mathrm{D}}$ receptor. The mode of action of the triptans is hypothesized to be three-fold 6 :

1. Binding of postsynaptic vascular $5-\mathrm{HT}_{1 \mathrm{~B}}$ receptors, to stimulate vasoconstriction of meningeal vessels.

2. Binding of presynaptic neuronal 5-HT $\mathrm{HD}_{1 \mathrm{D}}$ receptors, to inhibit release of proinflammatory neuropeptides.

3. Binding of presynaptic neuronal $5-\mathrm{HT}_{1 \mathrm{D}}$ receptors, to diminish the firing rate in trigeminal neurons and the trigeminal nucleus caudalis (central action).

The question of central absorption of sumatriptan has always been problematic and subsequent drug developments have suggested that central action may be important; this is, however, still controversial.

\section{Pharmacokinetics (Table 1)}

Subcutaneous sumatriptan has the best bioavailability and most rapid absorption of all the triptans. However, the bioavailability of oral and intranasal sumatriptan is low, primarily due to first pass metabolism and partly due to incomplete absorption. ${ }^{7}$ The newer triptans all have better oral bioavailability than sumatriptan, with naratriptan having the greatest bioavailability. ${ }^{8-10}$ Whether better oral bioavailability translates into a more consistent clinical response has not been determined. The presence of gastric stasis, which frequently accompanies a migraine attack, may delay the absorption of oral medications. ${ }^{11}$ The time to reach peak concentration $\left(\mathrm{T}_{\max }\right)$ is significantly increased during an attack with oral zolmitriptan, ${ }^{12}$ but not with oral sumatriptan ${ }^{7}$ or rizatriptan. ${ }^{10}$ Rizatriptan (oral tablet) reaches maximum serum levels within 1-1.5 hours during an attack. Food delays absorption of the oral tablet by about one hour. Rizatriptan wafer is a freeze-dried formulation, which rapidly disintegrates on the tongue (within seconds), is swallowed with saliva and absorbed from the gastrointestinal tract (i.e., not sublingual); absorption is somewhat slower compared to the oral tablet. ${ }^{10}$ Naratriptan has the longest elimination half-life and hence, the longest duration of action of the triptans. ${ }^{8}$ Naratriptan, rizatriptan and zolmitriptan are all more lipophilic (i.e., more likely to cross the blood-brain barrier) than sumatriptan; however, the clinical significance of differences in lipophilicity is not yet clear. ${ }^{11}$

All triptans are metabolized in the liver. ${ }^{7-10}$ Sumatriptan is metabolized by monoamine oxidase A (MAO-A), which is found in the liver and gastrointestinal tract. ${ }^{7}$ Naratriptan is metabolized by cytochrome P-450 CYP-450) enzymes (exact isoenzymes are not known). ${ }^{8}$ The metabolism of zolmitriptan involves both cytochrome P-450 (1A2 isoenzyme (CYP1A2)) and MAO-A. Zolmitriptan is metabolized to an active $\mathrm{N}$-desmethyl metabolite, which is two to six times more potent than the parent compound and may contribute to the overall efficacy. ${ }^{9,12}$ Rizatriptan is primarily metabolized by MAO-Ato inactive metabolites and an active $\mathrm{N}$-monodesmethyl metabolite (with activity similar to that of the parent compound). ${ }^{10,13}$

\section{DRUG INTERACTIONS (Table 2)}

\section{Monoamine oxidase inhibitors (MAOIs)}

Since sumatriptan, rizatriptan and zolmitriptan are metabolized by MAO-A, drugs that inhibit MAO-Aare likely to interact with these triptans. Selective MAO-A inhibitors (e.g., moclobemide) or nonselective MAOIs (e.g., phenelzine, tranylcypromine) may inhibit the metabolism of these triptans, resulting in increased plasma concentrations. MAOIs also inhibit the degredation of serotonin, thereby increasing the concentration of serotonin in the synapse. As a result, serotonin syndrome may potentially occur with concomitant use of MAOIs and triptans (see discussion below on serotonin syndrome). The concurrent administration, or use of sumatriptan, rizatriptan or zolmitriptan within two weeks of discontinuation of MAOI therapy is contraindicated. ${ }^{7,9,10,14}$ Since naratriptan is not metabolized by MAO-A, its use is not contraindicated with MAOIs (but there is a theoretical possibility of serotonin syndrome - see below).

\section{Ergot-containing drugs and other 5-HT 1 agonists}

Due to the theoretical possibility of additive vasospastic reactions (including coronary vasospasm), manufacturers of triptans contraindicate their use within 24 hours before, or after, treatment with ergot-containing drugs or their derivatives (e.g., ergotamine, dihydroergotamine, methysergide) or other $5-\mathrm{HT}_{1}$ agonists (i.e., other triptans).$^{7-10}$ However, in clinical practice, neurologists sometimes prescribe triptans for patients who are receiving methysergide as prophylactic therapy; careful monitoring for adverse effects is advised in this situation. Different dosage forms of the same triptan can be taken within a 24-hour period, after an appropriate interval (generally two hours).

\section{Serotonergic drugs}

Since the triptans are serotonin receptor agonists, concurrent use with other serotonergic drugs [e.g., MAOIs, selective serotonin reuptake inhibitors (SSRIs), lithium] may result in serotonin syndrome. Features of the serotonin syndrome include: mental status and behavioural change (e.g., agitation, excitement, hypomania, obtundation), motor system involvement (e.g., myoclonus, hemiballismus, tremor, hyperreflexia, motor weakness, dysarthria, ataxia) and autonomic symptoms (e.g., fever, chills, diarrhea). Not all of these symptoms are necessarily present in each case. Serotonin syndrome can be relatively mild or, in rare cases, may be life-threatening. There have been some case reports of 'suspected'serotonin syndrome with sumatriptan used in combination with SSRIs or lithium; however, other reports have not noted adverse effects with these combinations. Since serotonin syndrome is, theoretically, possible with concurrent use of any of the triptans and other serotonergic drugs, such as SSRIs (e.g., fluoxetine, fluvoxamine, paroxetine, sertraline, citalopram) or lithium, caution is advised. ${ }^{15,16}$ In clinical practice, triptans are usually well-tolerated in patients on SSRI therapy. ${ }^{13,17}$ Tricyclic antidepressants, venlafaxine, 
Table 1: Pharmacokinetics of 5-HT ${ }_{1 \mathrm{~B} / 1 \mathrm{D}}$ Receptor Agonists ${ }^{7-12}$

\begin{tabular}{|c|c|c|c|c|c|c|}
\hline Drug & Bioavailability & $\begin{array}{l}T_{\max } \text { outside } \\
\text { of attack }\end{array}$ & $\begin{array}{l}T_{\text {max }} \text { during } \\
\text { attack }\end{array}$ & $\begin{array}{l}\text { Elimination } \\
\text { half-life }\end{array}$ & Metabolism & Elimination \\
\hline Naratriptan & $\begin{array}{l}74 \% \text { (females) } \\
63 \% \text { (males) }\end{array}$ & $2-5 \mathrm{~h}$ & $3.8 \mathrm{~h}$ & $5-8 \mathrm{~h}$ & $\begin{array}{l}\text { CYP450 } \\
\text { (various isoenzymes) }\end{array}$ & $\begin{array}{l}\text { urine: } 50 \% \text { unchanged } \\
30 \% \text { metabolites (inactive) }\end{array}$ \\
\hline Rizatriptan & $45 \%$ & $\begin{array}{l}1-1.5 \mathrm{~h} \text { (oral tablets) } \dagger \\
1.6-2.5 \mathrm{~h} \\
\text { (disintegrating tablets) }\end{array}$ & $\begin{array}{l}\text { Same as outside } \\
\text { of attack }\end{array}$ & $2-3 \mathrm{~h}$ & MAO-A & $\begin{array}{l}\text { urine: } 8-16 \% \text { unchanged } \\
51 \% \text { metabolites } \\
\text { (inactive \& active } \mathrm{N}- \\
\text { monodesmethyl) }\end{array}$ \\
\hline Sumatriptan & $\begin{array}{l}\text { SC: } 96 \% \\
\text { Oral: } 14 \% \\
\text { Nasal: } 16 \%\end{array}$ & $\begin{array}{l}\mathrm{SC}: 15 \mathrm{~min} \\
\text { oral }(100 \mathrm{mg}): 0.5-5 \mathrm{~h}^{*} \\
\text { nasal: } 1-1.5 \mathrm{~h}\end{array}$ & $\begin{array}{l}\text { SC: ? } \\
\text { oral: } 2 \mathrm{~h} \\
\text { nasal: ? }\end{array}$ & $2 \mathrm{~h}$ & MAO-A & $\begin{array}{l}80 \% \text { non-renal } \\
\text { urine: metabolites (inactive) }\end{array}$ \\
\hline Zolmitriptan & $40 \%$ & $2 \mathrm{~h}$ & $4 h^{12}$ & $\begin{array}{l}2.5-3 \mathrm{~h} \text { (parent) } \\
3 \mathrm{~h} \text { (metabolite) }\end{array}$ & $\begin{array}{l}\text { CYP-1A2 and } \\
\text { MAO-A }\end{array}$ & $\begin{array}{l}\text { feces: } 27 \% \text {; urine: } 64 \% \text { - } \\
8 \% \text { unchanged; rest as } \\
\text { metabolites (inactive \& } \\
\text { active N-desmethyl) }\end{array}$ \\
\hline
\end{tabular}

*70-80\% of maximum serum values $\left(\mathrm{C}_{\max }\right)$ are attained within 30-45 minutes of dosing $\dagger$ without food; absorption is delayed by one hour with food (tablets only)

$\mathrm{T}_{\max }=$ time to maximum serum concentration (note: onset of action may occur sooner)
$\mathrm{SC}=$ subcutaneous

CYP-450 = cytochrome P-450

MAO-A= monoamine oxidase $\mathrm{A}$

Table 2: Drug Interactions with 5-HT ${ }_{1 \mathrm{~B} / 1 \mathrm{D}}$ Receptor Agonists ${ }^{7-10,13-17}$

\begin{tabular}{|c|c|c|c|c|}
\hline & Naratriptan & Rizatriptan & Sumatriptan & Zolmitriptan \\
\hline $\begin{array}{l}\text { Monoamine oxidase } \\
\text { inhibitors (MAOIs)* }\end{array}$ & $\begin{array}{l}\text { MAOIs not } \\
\text { contraindicated, as } \\
\text { naratriptan is not } \\
\text { metabolized by MAO; } \\
\text { theoretical possibility of } \\
\text { SS (no reports) }\end{array}$ & $\begin{array}{l}\text { moclobemide - significant } \\
\uparrow \text { in rizatriptan AUC (by } \\
119 \% \text { ), } C_{\max } \text { (by } 41 \% \text { ) \& } \\
\text { AUC of active metabolite } \\
\text { (by }>400 \% \text { ) in } 12 \\
\text { volunteers; possibility of } \\
\text { SS - avoid use within } 14 \\
\text { days of MAOI } \\
\text { discontinuation }\end{array}$ & $\begin{array}{l}\text { inhibition of sumatriptan } \\
\text { metabolism \& } \uparrow \text { plasma } \\
\text { levels; possibility of SS - } \\
\text { avoid use within } 14 \text { days } \\
\text { of MAOI discontinuation }\end{array}$ & $\begin{array}{l}\text { moclobemide - } \uparrow \text { AUC \& } \\
\mathrm{C}_{\max } \text { for zolmitriptan (by } \\
26 \% \text { ) \& active metabolite } \\
\text { ( } 3 \text {-fold) - avoid use within } \\
14 \text { days of MAOI } \\
\text { discontinuation }\end{array}$ \\
\hline Ergot derivatives $\dagger$ & possibility of additive & possibility of additive & possibility of additive & possibility of additive \\
\hline Other $5-\mathrm{HT}_{1}$ agonists & $\begin{array}{l}\text { vasospasm - avoid use } \\
\text { within } 24 \mathrm{~h} \text { of each other }\end{array}$ & $\begin{array}{l}\text { vasospasm - avoid use } \\
\text { within } 24 \mathrm{~h} \text { of each other }\end{array}$ & $\begin{array}{l}\text { vasospasm - avoid use } \\
\text { within } 24 \mathrm{~h} \text { of each other }\end{array}$ & $\begin{array}{l}\text { vasospasm - avoid use } \\
\text { within } 24 \mathrm{~h} \text { of each other }\end{array}$ \\
\hline $\begin{array}{l}\text { Serotonergic drugs (e.g., } \\
\text { SSRIs } \neq \text {, lithium) }\end{array}$ & $\begin{array}{l}\text { theoretical possibility of SS } \\
\text { (no reports) - monitor }\end{array}$ & $\begin{array}{l}\text { theoretical possibility of SS } \\
\text { (no reports) - monitor; no } \\
\text { interaction with paroxetine } \\
(20 \mathrm{mg} / \mathrm{d}) \text { in } 12 \text { volunteers }\end{array}$ & $\begin{array}{l}\text { possibility of SS (rare } \\
\text { reports in literature) - } \\
\text { monitor }\end{array}$ & $\begin{array}{l}\text { theoretical possibility of SS } \\
\text { (no reports) - monitor; no } \\
\text { interaction with fluoxetine } \\
(20 \mathrm{mg} / \mathrm{d}) \text { in } 16 \text { volunteers }\end{array}$ \\
\hline Beta-blockers & no reports of interaction & $\begin{array}{l}\text { propranolol }(240 \mathrm{mg} / \mathrm{d})-\uparrow \\
\text { AUC (by } 70 \%) \text { of } \\
\text { rizatriptan }(10 \mathrm{mg}) \text { in } 11 \\
\text { volunteers }- \text { use } 5 \mathrm{mg} \text { single } \\
\text { doses (max. } 10 \mathrm{mg} / \mathrm{d}) \text {; } \\
\text { nadolol and metoprolol - } \\
\text { no interaction in } 12 \\
\text { volunteers }\end{array}$ & $\begin{array}{l}\text { no reports of interaction; } \\
\text { no interaction with } \\
\text { propranolol }(160 \mathrm{mg} / \mathrm{d}) \& \\
\text { sumatriptan }(300 \mathrm{mg} \text { single } \\
\text { dose }) \text { in } 10 \text { volunteers }\end{array}$ & $\begin{array}{l}\text { propranolol }(160 \mathrm{mg} / \mathrm{d}) \\
1.5 \text {-fold } \uparrow \mathrm{AUC} \& \mathrm{C}_{\max } \text { of } \\
\text { zolmitriptan; } \downarrow \mathrm{C}_{\max } \text { (by } \\
30 \%) \& \text { AUC (by } 15 \%) \text { of } \\
\text { active metabolite; not } \\
\text { clinically significant; no } \\
\text { dose adjustments recom- } \\
\text { mended by manufacturer }\end{array}$ \\
\hline $\begin{array}{l}\text { Cimetidine \& other } \\
\text { CYP1A2 inhibitors (e.g., } \\
\text { fluvoxamine, } \\
\text { ciprofloxacin) }\end{array}$ & no reports of interaction & no reports of interaction & no reports of interaction & $\begin{array}{l}\text { cimetidine }-2 \text {-fold } \uparrow \text { half- } \\
\text { life \& AUC of zolmitriptan } \\
\& \text { active metabolite; do not } \\
\text { exceed } 5 \mathrm{mg} / 24 \mathrm{~h} \text { of } \\
\text { zolmitriptan }\end{array}$ \\
\hline
\end{tabular}

*MAOIs include selective MAO-Ainhibitors (i.e., moclobemide) and non-selective MAOIs (e.g., phenelzine, tranylcypromine)

$\dagger$ Ergot derivatives include ergotamine, dihydroergotamine (DHE), methysergide

$\ddagger$ SSRIs include fluoxetine, fluvoxamine, paroxetine, sertraline, citalopram; interaction is unlikely with tricyclic antidepressants, venlafaxine, nefazodone or bupropion

$\mathrm{SS}=$ serotonin syndrome; $\mathrm{AUC}=$ area under the plasma-concentration-time curve; $\mathrm{C}_{\max }=$ maximum plasma concentration; $\mathrm{BP}=$ blood pressure 
Table 3: Summary of randomized placebo-controlled trials (combined data): headache response at one and two hours

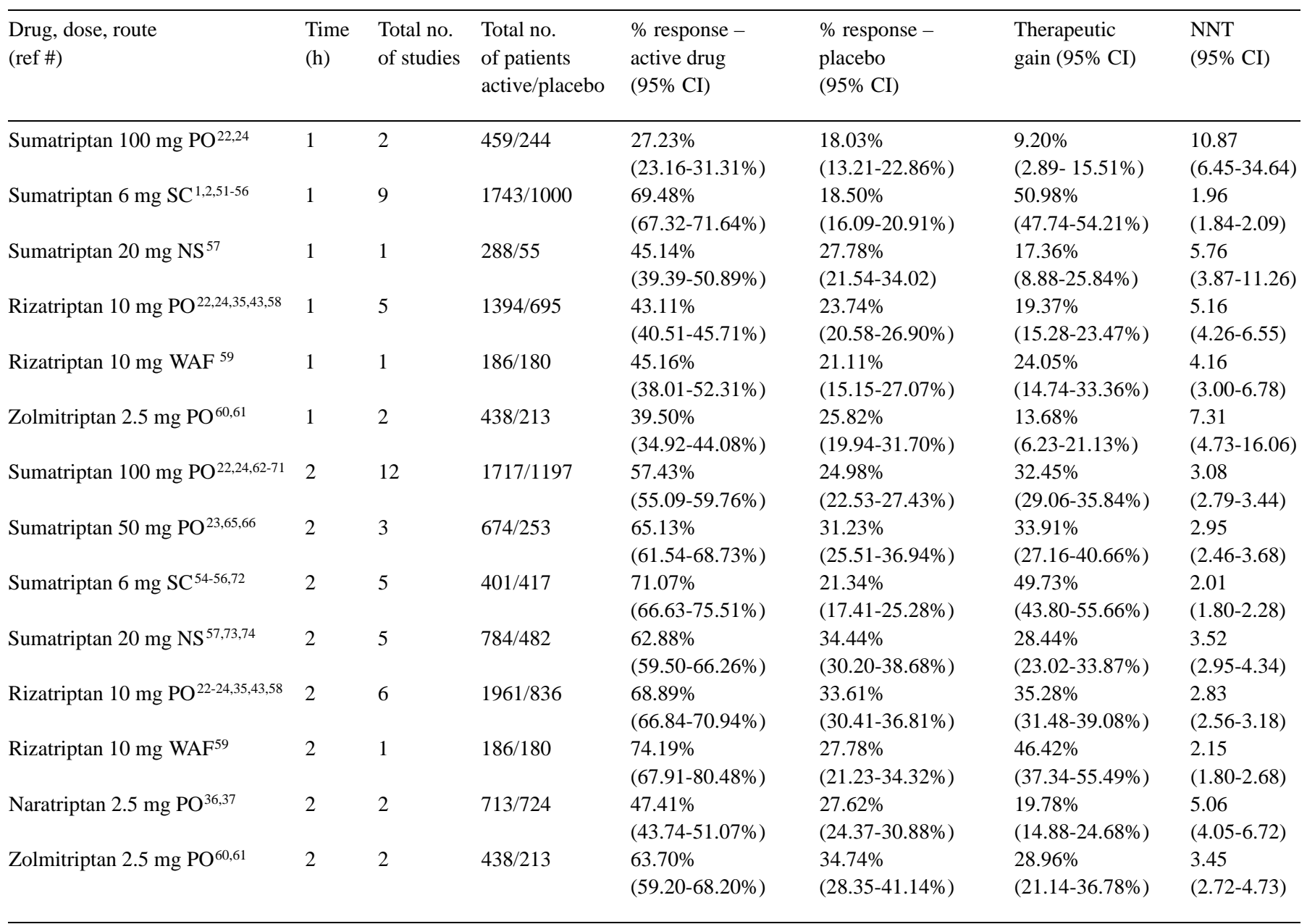

$\mathrm{PO}=$ oral; $\mathrm{SC}=$ subcutaneous; $\mathrm{NS}$ = nasal spray; WAF = wafer; $\mathrm{CI}=$ confidence interval; $\mathrm{NNT}=$ number needed to treat

nefazodone or bupropion are unlikely to result in serotonin syndrome when used in combination with triptans. ${ }^{15}$

\section{Beta-blockers}

Concomitant use of propranolol has resulted in increased bioavailability and serum concentrations of rizatriptan ${ }^{10}$ and zolmitriptan. ${ }^{14,18}$ The interaction with zolmitriptan is not considered to be clinically significant and no dosage adjustments are necessary. ${ }^{9}$ For rizatriptan, use of $5 \mathrm{mg}$ as a single dose (maximum $10 \mathrm{mg} / \mathrm{d}$ ) is recommended with concomitant propranolol therapy; however, no interactions have been noted with concomitant use of nadolol or metoprolol. ${ }^{10}$ Sumatriptan and naratriptan have not been reported to interact with propranolol. 8,19

\section{Cimetidine}

Concomitant use of cimetidine has been found to increase the half-life and bioavailability of zolmitriptan and its active metabolite. Therefore, a total dose of $5 \mathrm{mg}$ in a 24-hour period should not be exceeded. This recommendation also applies to concomitant use of zolmitriptan with other CYP1A2 inhibitors (e.g., fluvoxamine, ciprofloxacin), since a similar interaction may occur, theoretically. ${ }^{9}$ Cimetidine has not been reported to interact with the other triptans.

\section{EFFICACY RESULTS}

\section{a) Randomized placebo-controlled trials (Tables 3-5)}

Headache response rates at one and two hours are presented in Table 3. The lowest NNT for headache response at one and two hours is observed with subcutaneous sumatriptan (1.96 and 2.01, respectively). Among the oral formulations, the lowest NNT for headache response at two hours is observed with rizatriptan wafer $(2.15$, based on one trial). Naratriptan has the highest NNT (5.06) at two hours. However, differences among most of the oral/intranasal triptans are relatively small at two hours.

Pain-free rates at one and two hours are presented in Table 4. The lowest NNT for pain-free at one and two hours is observed 
Table 4: Summary of randomized, placebo-controlled trials (combined data): pain-free at one and two hours

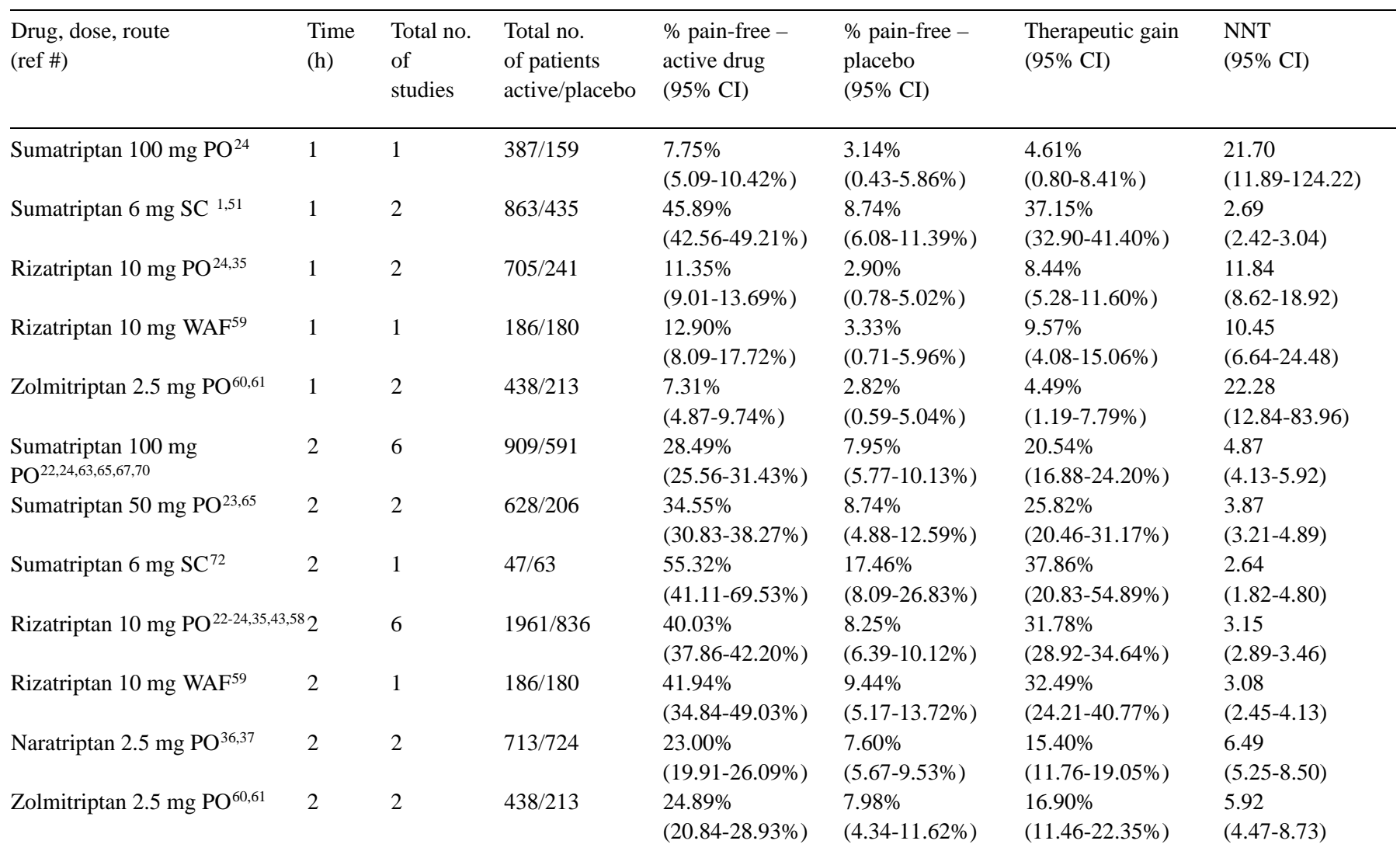

$\mathrm{PO}=$ oral; $\mathrm{SC}=$ subcutaneous; $\mathrm{NS}$ = nasal spray; $\mathrm{WAF}=$ wafer CI = confidence interval; $\mathrm{NNT}=$ number needed to treat

Table 5: Clinical disability score of $0 / 1$ at two hours (combined data) - randomized, placebo-controlled trials

\begin{tabular}{|c|c|c|c|c|c|c|}
\hline $\begin{array}{l}\text { Drug, dose, route } \\
\text { (ref \#) }\end{array}$ & $\begin{array}{l}\text { Total no. } \\
\text { of studies }\end{array}$ & $\begin{array}{l}\text { Total no. } \\
\text { of patients } \\
\text { active/placebo }\end{array}$ & $\begin{array}{l}\% \text { no clinical } \\
\text { disability - } \\
\text { active drug } \\
(95 \% \mathrm{CI})\end{array}$ & $\begin{array}{l}\% \text { no clinical } \\
\text { disability - } \\
\text { placebo } \\
(95 \% \mathrm{CI})\end{array}$ & $\begin{array}{l}\text { Therapeutic gain } \\
(95 \% \mathrm{CI})\end{array}$ & $\begin{array}{l}\text { NNT } \\
(95 \% \text { CI })\end{array}$ \\
\hline $\begin{array}{l}\text { Sumatriptan } 100 \mathrm{mg} \\
\mathrm{PO}^{22,24,34,65,66,71}\end{array}$ & 6 & $826 / 443$ & $\begin{array}{l}37.65 \% \\
(34.35-40.96 \%)\end{array}$ & $\begin{array}{l}19.19 \% \\
(15.52-22.85 \%)\end{array}$ & $\begin{array}{l}18.46 \% \\
(13.53-23.40 \%)\end{array}$ & $\begin{array}{l}5.42 \\
(4.27-7.39)\end{array}$ \\
\hline Sumatriptan $50 \mathrm{mg} \mathrm{PO} \mathrm{PO}^{23,34,65,66}$ & 4 & $931 / 340$ & $\begin{array}{l}42.32 \% \\
(39.15-45.49 \%)\end{array}$ & $\begin{array}{l}23.53 \% \\
(19.02-28.04 \%)\end{array}$ & $\begin{array}{l}18.79 \% \\
(13.28-24.30 \%)\end{array}$ & $\begin{array}{l}5.32 \\
(4.11-7.53)\end{array}$ \\
\hline Sumatriptan 6 mg SC 55,72 & 3 & $126 / 142$ & $\begin{array}{l}72.22 \% \\
(64.40-80.04 \%)\end{array}$ & $\begin{array}{l}29.58 \% \\
(22.07-37.08 \%)\end{array}$ & $\begin{array}{l}42.64 \% \\
(31.80-53.49 \%)\end{array}$ & $\begin{array}{l}2.34 \\
(1.87-3.14)\end{array}$ \\
\hline Sumatriptan 20 mg NS ${ }^{57,74}$ & 3 & $705 / 411$ & $\begin{array}{l}72.06 \% \\
(68.74-75.37 \%)\end{array}$ & $\begin{array}{l}51.82 \% \\
(46.99-56.66 \%)\end{array}$ & $\begin{array}{l}20.23 \% \\
(14.37-26.09 \%)\end{array}$ & $\begin{array}{l}4.94 \\
(3.83-6.96)\end{array}$ \\
\hline Rizatriptan 10 mg PO $22-24,35,58$ & 5 & $1506 / 534$ & $\begin{array}{l}43.56 \% \\
(41.05-46.06 \%)\end{array}$ & $\begin{array}{l}16.67 \% \\
(13.51-19.83 \%)\end{array}$ & $\begin{array}{l}26.89 \% \\
(22.86-30.93 \%)\end{array}$ & $\begin{array}{l}3.72 \\
(3.23-4.37)\end{array}$ \\
\hline
\end{tabular}

$\mathrm{PO}=$ oral SC $=$ subcutaneous $; \mathrm{NS}=$ nasal WAF $=$ wafer $; \mathrm{CI}=$ confidence interval; $\mathrm{NNT}=$ number needed to treat 
Table 6: Overall incidence of all adverse events (combined data) - randomized placebo-controlled trials

\begin{tabular}{|c|c|c|c|c|c|c|}
\hline $\begin{array}{l}\text { Drug, dose, route } \\
\text { (ref \#) }\end{array}$ & $\begin{array}{l}\text { Total no. } \\
\text { of studies }\end{array}$ & $\begin{array}{l}\text { Total no. } \\
\text { of patients } \\
\text { active/placebo }\end{array}$ & $\begin{array}{l}\% \text { adverse } \\
\text { events - } \\
\text { active drug } \\
(95 \% \mathrm{CI})\end{array}$ & $\begin{array}{l}\% \text { adverse } \\
\text { events - } \\
\text { placebo } \\
(95 \% \mathrm{CI})\end{array}$ & $\begin{array}{l}\text { Therapeutic } \\
\text { penalty } \\
(95 \% \mathrm{CI})\end{array}$ & $\begin{array}{l}\text { NNH } \\
(95 \% \mathrm{CI})\end{array}$ \\
\hline $\begin{array}{l}\text { Sumatriptan } 100 \mathrm{mg} \\
\mathrm{PO}^{22,24,34,62,63,65-69,71}\end{array}$ & 11 & $2715 / 1195$ & $\begin{array}{l}47.33 \% \\
(45.45-49.21 \%)\end{array}$ & $\begin{array}{l}33.31 \% \\
(30.63-35.98 \%)\end{array}$ & $\begin{array}{l}14.02 \% \\
(10.76-17.29 \%)\end{array}$ & $\begin{array}{l}7.13 \\
(5.78-9.30)\end{array}$ \\
\hline Sumatriptan $50 \mathrm{mg} \mathrm{PO} \mathrm{PO}^{23,65,66}$ & 3 & $399 / 254$ & $\begin{array}{l}48.37 \% \\
(43.47-53.27 \%)\end{array}$ & $\begin{array}{l}44.09 \% \\
(37.99-50.20 \%)\end{array}$ & $\begin{array}{l}4.28 \% \\
(-3.55-12.11 \%)\end{array}$ & $\begin{array}{l}23.38 \\
(8.26--28.13)\end{array}$ \\
\hline Sumatriptan 6 mg SC ${ }^{1,51,52,54,72}$ & 5 & $1098 / 580$ & $\begin{array}{l}70.58 \% \\
(67.89-73.28 \%)\end{array}$ & $\begin{array}{l}39.83 \% \\
(35.84-43.81 \%)\end{array}$ & $\begin{array}{l}30.76 \% \\
(25.95-35.57 \%)\end{array}$ & $\begin{array}{l}3.25 \\
(2.81-3.85)\end{array}$ \\
\hline Rizatriptan 10 mg PO $22-24,43$ & 4 & $1196 / 620$ & $\begin{array}{l}42.64 \% \\
(39.84-45.45 \%)\end{array}$ & $\begin{array}{l}28.55 \% \\
(24.99-32.10 \%)\end{array}$ & $\begin{array}{l}14.09 \% \\
(9.57-18.62 \%)\end{array}$ & $\begin{array}{l}7.10 \\
(5.37-10.45)\end{array}$ \\
\hline Naratriptan 2.5 mg PO ${ }^{36,37}$ & 2 & $537 / 551$ & $\begin{array}{l}31.47 \% \\
(27.54-35.40 \%)\end{array}$ & $\begin{array}{l}32.49 \% \\
(28.58-36.40 \%)\end{array}$ & $\begin{array}{l}-1.02 \% \\
(-6.56-4.53 \%)\end{array}$ & $\begin{array}{l}-98.50 \\
(22.09--15.25)\end{array}$ \\
\hline
\end{tabular}

$\mathrm{PO}=$ oral SC = subcutaneous; $\mathrm{CI}=$ confidence interval; $\mathrm{NNH}=$ number needed to harm

with subcutaneous sumatriptan (2.69 and 2.64, respectively). Among the oral triptans, the lowest NNT for pain-free at two hours is observed with rizatriptan wafer (3.08, based on one trial) and rizatriptan oral tablet (3.15). Naratriptan has the highest NNT (6.49) at two hours. As for response rates, differences among most of the oral/intranasal triptans are relatively small at two hours.

For a clinical disability score $0 / 1$ at two hours (Table 5), the lowest combined NNT is observed with subcutaneous sumatriptan (2.34). Among the oral formulations, the lowest combined NNT is observed with rizatriptan wafer (3.15, based on one trial) and rizatriptan oral tablet (3.72).

\section{b) Direct comparative trials}

\section{Zolmitriptan vs Sumatriptan}

Zolmitriptan (2.5 $\mathrm{mg}$ and $5 \mathrm{mg}$ ) has been compared to sumatriptan $(25 \mathrm{mg}$ and $50 \mathrm{mg}$ ) oral tablets in a randomized, double-blind, parallel-group study $(\mathrm{N}=1212)$. In this comparison, zolmitriptan $2.5 \mathrm{mg}$ was significantly more effective than the sumatriptan 25 and $50 \mathrm{mg}$ at two and four hours, in terms of headache response. Zolmitriptan $5 \mathrm{mg}$ was better than sumatriptan $25 \mathrm{mg}$ at all time points, and against sumatriptan $50 \mathrm{mg}$ at one hour and four hours. Pain relief over 24 hours was also assessed; both doses of zolmitriptan showed significantly better pain relief for up to six attacks treated, than either dose of sumatriptan. ${ }^{20}$

\section{Rizatriptan vs Naratriptan}

Rizatriptan (10mg) has been compared to naratriptan $(2.5 \mathrm{mg})$ $\mathrm{mg}$ in a randomized, double-blind, double-dummy, placebocontrolled study ( $\mathrm{N}=522)$. Rizatriptan was more effective than naratriptan and provided earlier headache relief (as early as 30 minutes). Significantly more patients were pain-free at two hours with rizatriptan than with naratriptan. Rizatriptan also provided earlier relief of associated migraine symptoms (i.e., nausea, photophobia, phonophobia) within two hours and more patients had normal functioning at two hours, compared to those on naratriptan. Patients on rizatriptan were more satisfied with their medication at two hours. However, there was no significant difference at 24 hours, in terms of overall treatment effect. ${ }^{21}$

\section{Rizatriptan vs Sumatriptan}

An early, randomized, double-blind, parallel-group, doseranging study $(\mathrm{N}=449)$ compared rizatriptan $10 \mathrm{mg}, 20 \mathrm{mg}$ and $40 \mathrm{mg}$ to placebo and to sumatriptan $100 \mathrm{mg}$ (rizatriptan $20 \mathrm{mg}$ and $40 \mathrm{mg}$ are not currently available in Canada). In terms of pain relief, rizatriptan $10 \mathrm{mg}$ was not significantly different from sumatriptan $100 \mathrm{mg}$ at one and two hours. ${ }^{22}$

Rizatriptan (5 $\mathrm{mg}$ and $10 \mathrm{mg}$ ) has been compared to sumatriptan $(25 \mathrm{mg}$ and $50 \mathrm{mg}$ ) and placebo in a randomized, double-blind, crossover study ( $\mathrm{N}=1329)$. Rizatriptan $5 \mathrm{mg}$ was superior to sumatriptan $25 \mathrm{mg}$ for pain relief at one and two hours. Rizatriptan $10 \mathrm{mg}$ was superior to sumatriptan for 'painfree' at one hour and four hours. Sumatriptan $25 \mathrm{mg}$ was superior to rizatriptan $5 \mathrm{mg}$ at 0.5 hours. Rizatriptan $(5 / 10 \mathrm{mg})$ resulted in a greater reduction in the associated symptom of nausea, compared to sumatriptan $(25 / 50 \mathrm{mg})$. Functional disability results showed that rizatriptan $5 \mathrm{mg}$ was superior to sumatriptan $25 \mathrm{mg}$ at 0.5 and 1.5 hours, while rizatriptan $10 \mathrm{mg}$ was superior to sumatriptan $50 \mathrm{mg}$ at three hours and four hours. Patients experienced more satisfaction with rizatriptan $10 \mathrm{mg}$ versus sumatriptan $50 \mathrm{mg}$ at two hours and four hours. No difference was detected between rizatriptan $5 \mathrm{mg}$ and sumatriptan $25 \mathrm{mg}$ in terms of patient satisfaction. ${ }^{23}$

A randomized, double-blind, triple-dummy, parallel-group trial $(\mathrm{N}=1091)$ compared rizatriptan $(5 \mathrm{mg}$ and $10 \mathrm{mg})$ and sumatriptan (100 mg) and placebo. Results were age-adjusted, since the rizatriptan group was younger than the sumatriptan group. Rizatriptan $10 \mathrm{mg}$ had a faster time to pain relief through 
two hours than sumatriptan $100 \mathrm{mg}$, demonstrating significantly more effect at one hour. Rizatriptan $10 \mathrm{mg}$ was superior to sumatriptan $100 \mathrm{mg}$ for pain-free at two hours. Compared to the sumatriptan group, significantly more patients in the rizatriptan $10 \mathrm{mg}$ group were without nausea at all time points through two hours. More patients showed improvement in functional disability at one hour and two hours with rizatriptan $10 \mathrm{mg}$. Adverse events were less frequent with rizatriptan $5 \mathrm{mg}$ (i.e., chest pain, any adverse event and any drug-related adverse event) compared to sumatriptan $100 \mathrm{mg}$. Rizatriptan $10 \mathrm{mg}$ showed fewer drug-related adverse events compared to sumatriptan $100 \mathrm{mg}^{24}$

\section{RELIEF OF ASSOCIATED SYMPTOMS}

The associated symptoms of migraine (i.e., nausea, vomiting, photophobia, phonophobia) contribute to migraine-related disability. Therefore, relief of associated symptoms is important in the overall management of migraine. The most distressing symptoms are gastrointestinal disturbances, which can be severe and incapacitating. During migraine attacks, nausea and anorexia occur in a significant proportion of patients; vomiting occurs less frequently. Approximately $30 \%$ of patients indicate that nausea interferes with their ability to take oral migraine medications. ${ }^{25}$ Based on data from direct comparative trials (see above), rizatriptan appears to provide earlier and better relief of migraine-associated nausea than sumatriptan ${ }^{23,24}$ or naratriptan. ${ }^{21}$

\section{CONSISTENCY OF RESPONSE}

The measurement of consistency of response is sometimes difficult since, in clinical practice, patients treat large numbers of attacks. The reasons for lack of response in some attacks are not known. Consistency can be measured in different ways: by utilizing group data (a number of patients treat a large number of attacks) and determining the percentage of responders at each headache treatment or by determining response rates in individual patients treating a number of attacks. Utilizing group data has a disadvantage, since patients who do not respond will drop out eventually and the process will select consistent responders. Determining consistency of response in individual patients is more clinically meaningful. Consistency studies have been published using group data for all the triptans, ${ }^{26-33}$ and using individual (intra-patient) data for $\operatorname{sumatriptan}^{34}$ and rizatriptan. ${ }^{35}$

\section{AdVERSE EVENTS/SAFETY}

The combined NNH for overall incidence of adverse events is shown in Table 6. The lowest combined NNH occurs with subcutaneous sumatriptan (3.25). Oral rizatriptan and oral sumatriptan $(100 \mathrm{mg})$ have similar combined $\mathrm{NNH}$ for overall incidence of adverse events (7.10 and 7.13, respectively). The results for oral zolmitriptan and oral naratriptan are inconsistent, with very large confidence intervals.

\section{Triptan symptoms/chest symptoms}

Naratriptan appears to have the best tolerability profile of the triptans, having a low incidence (similar to that of placebo) of triptan-type sensations (e.g., tightness of jaw/neck/chest, chest discomfort, paresthesias, flushing, heat sensations, dizziness, somnolence) ${ }^{36,37}$ It should be emphasized that while all triptans are generally well-tolerated, this must not be confused with safety. Since $5-\mathrm{HT}_{1 \mathrm{~B}}$ receptors are found on human coronary arteries, triptans have the potential to constrict coronary vessels; this constriction is usually insignificant in patients without underlying coronary heart disease. 'Chest symptoms'associated with the triptans are often described as a tightness, squeezing, heaviness or pain. Sometimes the pain extends to, or is confined to, the throat. These symptoms are not generally due to coronary vasoconstriction, since electrocardiographic abnormalities are rare. However, there have been reported cases of myocardial infarction with sumatriptan (available on market for the longest time) but not with the other three triptans, to date. Nevertheless, considering the large total number of doses taken, the absolute risk of myocardial infarction appears to be very small with sumatriptan or other triptans. ${ }^{38}$ All available triptans have a similar potential to cause coronary vasoconstriction ${ }^{39}$ and, therefore, should be avoided in patients with underlying cardiovascular or cerebrovascular diseases or those who have risk factors for such diseases, or in patients with uncontrolled hypertension. ${ }^{7-10}$

\section{Sulfonamide allergy}

Sumatriptan and naratriptan contain sulfonamide moieties, while zolmitriptan and rizatriptan do not. Sumatriptan and naratriptan should be used with caution in patients who have a history of sulfonamide (sulfa) allergy; risks and benefits should be assessed. ${ }^{7-10}$ However, sumatriptan and naratriptan are structurally different from the sulfonamide antimicrobial agents; the actual incidence of cross-reactivity with sulfonamides is not presently known.

\section{Pregnancy/lactation}

Interim results from an ongoing sumatriptan/naratriptan pregnancy registry (Glaxo Wellcome) are insufficient for drawing conclusions about the safety of sumatriptan use in pregnancy. The birth defect rate for sumatriptan users does not appear to differ from the expected rate of $3 \%$ (2-5\%); no pattern has been observed in reported defects, to date. ${ }^{40}$ Another population-based, retrospective study $(\mathrm{N}=34)$ found that sumatriptan exposure during pregnancy was associated with an increased risk of preterm delivery and low birth weight. ${ }^{41}$ To date, no data are available for naratriptan, zolmitriptan or rizatriptan use during pregnancy. The manufacturers of triptans do not recommend their use during pregnancy. ${ }^{7-10}$

Sumatriptan is excreted in breast milk; the estimated amount is $3.5 \%$ of the dose taken by the mother. If breast milk is discarded for eight hours after a dose, a negligible amount would be present in the breast milk. ${ }^{42}$ However, the manufacturer recommends avoiding breastfeeding for 24 hours after a dose of sumatriptan. ${ }^{7}$ No data are available on breast milk excretion for naratriptan, zolmitriptan or rizatriptan; the manufacturers advise caution. ${ }^{8-10}$

\section{HEADACHE RECURRENCE}

Recurrence is the return of a headache to moderate or severe intensity within 24 hours, after it has become mild or absent (pain-free) in the first two hours. After a two-hour response, the 
Table 7: Recommended doses and availability ${ }^{7-10}$

\begin{tabular}{|c|c|c|}
\hline Drug & Recommended Doses & Availability in Canada \\
\hline Naratriptan (Amerge ${ }^{\circledR}-$ Glaxo Wellcome) & $\begin{array}{l}1 \mathrm{mg} \text { or } 2.5 \mathrm{mg} \text { (optimal dose); may repeat } \\
\text { once after } 4 \text { hours; max. } 5 \mathrm{mg} / 24 \text {-hour period }\end{array}$ & Tablets: $1 \mathrm{mg}, 2.5 \mathrm{mg}$ \\
\hline Rizatriptan (Maxalt ${ }^{\mathrm{TM}}$, Maxalt RPD ${ }^{\mathrm{TM}}{ }_{-}$ & $5 \mathrm{mg}$ or $10 \mathrm{mg}$ (optimal dose); may repeat & Tablets: $5 \mathrm{mg}, 10 \mathrm{mg}$ \\
\hline Merck Frosst) & $\begin{array}{l}\text { after } 2 \text { hours; max. } 20 \mathrm{mg} / 24 \text {-hour period } \\
\text { With propranolol: } 5 \mathrm{mg} \text { single dose; } \\
\max .10 \mathrm{mg} / 24 \text {-hour period }\end{array}$ & $\begin{array}{l}\text { Orally-disintegrating tablets } \\
\text { (wafers - RPD`): } 5 \mathrm{mg}, 10 \mathrm{mg}\end{array}$ \\
\hline \multirow[t]{3}{*}{ Sumatriptan (Imitrex ${ }^{\circledR}-$ Glaxo Wellcome) } & $\begin{array}{l}\text { Oral: } 25 \mathrm{mg}, 50 \mathrm{mg} \text { (optimal dose) or } 100 \mathrm{mg} \text {; } \\
\text { may repeat after } 2 \text { hours; max. } 200 \mathrm{mg} / 24 \text {-hour period) }\end{array}$ & Tablets: $25 \mathrm{mg}, 50 \mathrm{mg}, 100 \mathrm{mg}$ \\
\hline & $\begin{array}{l}\text { Nasal: } 5 \mathrm{mg} \text { or } 20 \mathrm{mg} \text { (optimal dose) in one } \\
\text { nostril; may repeat after } 2 \text { hours; } \\
\text { max. } 40 \mathrm{mg} / 24 \text {-hour period }\end{array}$ & Nasal Spray: $5 \mathrm{mg}, 20 \mathrm{mg}$ \\
\hline & $\begin{array}{l}\mathrm{SC}: 6 \mathrm{mg} \text {; may repeat in one hour; } \\
\max .12 \mathrm{mg} / 24 \text {-hour period }\end{array}$ & SC Injection: $6 \mathrm{mg}$ (auto-injector) \\
\hline Zolmitriptan (Zomig ${ }^{\circledR}$ - AstraZeneca) & $\begin{array}{l}2.5 \mathrm{mg} \text { (optimal dose) or } 1 \mathrm{mg} * \\
\text { (break } 2.5 \mathrm{mg} \text { tablet in half for approximate } \\
\text { dose); may repeat after } 2 \text { hours; } \\
\text { max. } 10 \mathrm{mg} / 24 \text {-hour period }\end{array}$ & $\begin{array}{l}\text { Tablets: } 2.5 \mathrm{mg} \\
\text { (orally-disintegrating tablets, } \\
\text { nasal spray not currently } \\
\text { available in Canada) }\end{array}$ \\
\hline
\end{tabular}

$\mathrm{SC}=$ subcutaneous

* Although the manufacturer suggests a $1 \mathrm{mg}$ dose, there appears to be little clinical data to support this dose; in the U.S., a 5 mg dose has also been approved

recurrence rates range from $21-40 \%$ for sumatriptan, ${ }^{22-24,34,54,72}$ $35-47 \%$ for rizatriptan $22-24,35,43,58,59$ and $22-37 \%$ for zolmitriptan. ${ }^{30,44,45,60,61}$ Recurrence rates for naratriptan appear to be somewhat lower (17-28\%, after a four-hour response). ${ }^{46}$ The reason for the lower headache recurrence rate with naratriptan is unknown.

\section{RECOMMENDED DOSES AND AVAILABILITY ${ }^{7-10}$}

Recommended doses and availability are shown in Table 7.

\section{DISCUSSION}

The introduction of sumatriptan in the early 1990s revolutionized acute migraine therapy and resulted in an increased understanding of migraine pathophysiology. This review has attempted to consolidate evidence concerning efficacy and tolerability of the four available triptans. Data from placebo-controlled and direct comparative trials of triptans, which have been published in full (to date), have been utilized. Data from abstracts or posters presented at meetings has not been included since such data are difficult to evaluate. Direct comparative studies are best for comparing relative efficacy of medications. However, there are few direct comparative trials of triptans published in full. Therefore, data from placebocontrolled trials have been pooled and combined NNTs or NNHs have been calculated for each triptan (according to dosage form and strength).

Interpretation of relative NNT or NNH among the different triptans should be undertaken with caution, since trial designs and patient populations may differ. Conclusions regarding relative efficacy, based on pooled data from placebo-controlled trials, are difficult to make. For naratriptan, four-hour efficacy data is usually presented in trials, since it has a slow onset of action; however, four-hour data have not been included in this review.

Patient preference: What do patients want from their acute migraine medication? This has been reviewed by Silberstein. ${ }^{25}$ The top three were:

1. Provides quick headache relief

2. Decreases headache pain

3. Decreases likelihood of headache recurrence

Onset of headache relief: Subcutaneous sumatriptan has the most rapid onset of action. Among the oral triptans, rizatriptan appears to have the fastest onset.

Efficacy (headache pain reduction): Subcutaneous sumatriptan is more effective than the oral/intranasal triptans; it may be considered for patients who do not respond to oral/intranasal triptans. Among the oral triptans, rizatriptan appears to have the best efficacy; however, differences among the oral triptans (except for naratriptan) are not great. Although naratriptan appears to be less effective than the other triptans, it may be very effective for some patients. Triptans are effective when taken at any stage of a migraine attack; however, they appear to have greater efficacy when taken earlier in an attack.

Relief of associated symptoms of migraine, particularly nausea/vomiting, is also important. Rizatriptan appears to provide earlier and better relief of migraine-associated nausea than the other available triptans.

Does lack of response to one triptan predict lack of response to another? This would not seem to be the case. One open trial 
has quantified the chance of responding to zolmitriptan or rizatriptan, if there is no response to sumatriptan. ${ }^{47}$ At two hours, $73 \%$ (35 out of 48) of sumatriptan nonresponders experienced a headache response to zolmitriptan and $81 \%$ (39 out of 48) to rizatriptan. However, approximately 19\% (9 out of 48) of sumatriptan nonresponders did not respond to zolmitriptan or rizatripan. Further controlled studies are needed in this area.

Headache recurrence: Headache recurrence, following successful acute therapy, is problematic for about $40 \%$ of all migraineurs. All of the triptans are associated with headache recurrence. Since headache recurrence may be defined differently in clinical trials, it is difficult to compare recurrence rates based on different trials. Furthermore, patients could usually take analgesics two hours after the triptan dose, if the response to the triptan was inadequate; this may have affected reported recurrence rates in trials. Direct comparative trials are best for comparing headache recurrence rates. Headache recurrence occurs only after a headache response; therefore, it should be calculated as a percentage of total responders and not a percentage of total patients taking the drug (i.e., responders and nonresponders). Naratriptan appears to exhibit lower recurrence rates than the other available triptans. The reason for this is not clear and does not appear to be related to its longer half-life. Based on an evaluation of clinical parameters that may affect headache recurrence with naratriptan, lower recurrence rates were noted in patients who treated their attacks earlier (i.e., within three hours of onset) and in those obtaining complete relief (pain-free) within four hours postdose. ${ }^{46}$ Whether this also applies to other triptans remains to be established.

Consistency: Consistency in response is becoming more important as an efficacy endpoint. Studies evaluating consistency have been performed for all four drugs; however, care must be employed when interpreting the data. In some studies, patients treated a large number of attacks over many months (group data), which selected consistent responders sequentially. Consistency among the triptans should ideally be compared utilizing intra-patient data obtained from direct comparative clinical trials.

Tolerability/Safety: The matter of tolerability vs. safety is problematic. Safety is always a great concern; for a nonfatal condition such as migraine, a medication that causes serious adverse events is not appropriate. Recent reviews suggest that the triptans are generally safe, considering the large number of attacks treated, to date. The more frightening symptoms are those which involve 'chest sensations'; these sensations are general throughout the class. However, they do not seem to presage the onset of more serious cardiac problems.

Which triptan to start with? This is an extremely difficult question to answer; based on the randomized controlled trials available, there is no clear-cut winner in the group. A small advantage in rapidity of onset within a group may not be apparent in an individual. A small difference in the tolerability may not be apparent either. It is clear from all the studies that the most rapidly-acting and the most effective treatment is sumatriptan subcutaneous injection. It was the first to be licensed in Canada, yet experience has shown that many patients do not accept an injection as well as an oral dosage form (this does not include cluster headache patients, in whom this preparation is the most effective symptomatic treatment available for acute attacks of cluster). The intranasal route would seem intuitively to have a faster onset of action; however, results are inconsistent, possibly due to the fact that patients may not use the device properly or some of the medication may be swallowed. There may be a problem with bitter taste (e.g., sumatriptan nasal). The novel, orally-disintegrating form (wafer) of rizatriptan (RPD) appears to be more popular than the standard tablet form.

The characteristics of the headache (e.g., severity, time to peak intensity, degree of disability, time to disability) may be considered in selecting a particular triptan. If the headache has a rapid progression to severe intensity, with a high degree of associated disability, a rapid onset medication (e.g., subcutaneous sumatriptan) may be the best option. Oral rizatriptan also has a fairly rapid onset of action. A slowly developing headache, which has a long time course, may be treated by a triptan with relatively slow onset but longer duration of action (e.g., naratriptan). For patients experiencing nausea and vomiting with their attacks, nonoral routes of administration should be considered (e.g., subcutaneous, intranasal). Alternatively, an orally-disintegrating tablet/wafer may be considered for patients experiencing nausea without vomiting (rizatriptan wafer is absorbed in gastrointestinal tract). Patients who experience many triptan adverse events may be tried on naratriptan, which tends to have a milder adverse effect profile than the other triptans. Patient preference may also be considered. An orally-disintegrating tablet formulation (e.g., rizatriptan wafer) may be considered for patients who desire a discreet and convenient oral dosage form.

Frequent use and rebound headache: It must be remembered that the triptans are symptomatic medications, most of which have short half-lives. Some patients have found that they need to take them on a very frequent basis, having tried appropriate prophylactic medication in the past and failed. The question of whether this causes problems has been reviewed by Limmroth, ${ }^{48}$ who found that frequent usage of zolmitriptan and naratriptan may produce rebound headaches. Overuse of sumatriptan has also been reported to result in medication-induced/chronic headache. ${ }^{49,50}$ In the author's (MJG) experience, the use of a triptan for a number of years may lead to refractoriness. The headache may recur faster and the benefit tends to be less complete; this needs to be studied further. Manufacturers do not offer much guidance concerning maximum recommended usage over a longer period of time. Maximum doses per 24-hour period are generally stated but guidelines regarding maximum monthly usage are not provided. Many clinicians would set a limit of between 12 to 18 doses of a triptan per month; however, data supporting or refuting this recommendation are lacking.

\section{Conclusion}

In summary, the triptans are effective symptomatic medications for acute migraine attacks. They represent a great advance in migraine management and enhance the quality of life of migraineurs. Sumatriptan has the most extensive data supporting its efficacy, tolerability and safety. Newer triptans may have some advantages over sumatriptan. Although differences exist among the triptans, they appear to be relatively 
small. Each triptan is efficacious and each has its place. Individual patients may find one more effective and/or better tolerated than another. If a patient does not respond to, or tolerate, one triptan, others may be tried. However, it must be remembered that there still exists a sizable minority of patients in whom triptans are not effective or use is limited by adverse events. Selection of a suitable delivery system, based on the characteristics of an individual patient's attacks and/or patient preference, is also important. It is too soon to determine which triptan is best. Further direct comparative trials may assist in identifying patients that may benefit from a particular triptan.

\section{ACKNOWLedGeMenTs}

We thank the following participants of the consensus conference for their input: Michel Aubé, MD, McGill University, Montreal; Werner J. Becker, MD, University of Calgary, Calgary; John G. Edmeads, MD, University of Toronto, Toronto; Robert F. Nelson, MD, University of Ottawa, Ottawa; William E. M. Pryse-Phillips*, MD, Memorial University, Newfoundland; R. Allan Purdy, MD, Dalhousie University, Halifax; Gordon Robinson, MD, University of British Columbia, Vancouver; Janet Vickers, MD, The Credit Valley Hospital, Mississauga, Ont.

This publication was funded by an unrestricted educational grant from AstraZeneca Canada Inc., Glaxo Wellcome Inc., and Merck Frosst Canada Inc.

*William E. M. Pryse-Phillips has since become a consultant for Glaxo Wellcome Inc.

\section{REFERENCES}

1. Cady RK, Wendt JK, Kirchner JR, et al. Treatment of acute migraine with subcutaneous sumatriptan. JAMA1991;265:2831-2835.

2. The Subcutaneous Sumatriptan International Study Group. Treatment of migraine attacks with sumatriptan. N Engl J Med 1991;325:316-321.

3. Tfelt-Hansen P. Efficacy and adverse events of subcutaneous, oral, and intranasal sumatriptan used for migraine treatment: a systematic review based on number needed to treat. Cephalalgia 1998;18:532-538.

4. Pryse-Phillips WEM, Dodick DW, Edmeads JG, et al. Guidelines for the diagnosis and management of migraine in clinical practice. CMAJ 1997;156:1273-1287.

5. Pryse-Phillips WEM, Dodick DW, Edmeads JG, et al. Guidelines for the nonpharmacological management of migraine in clinical practice. CMAJ 1998;159:47-54.

6. Hargreaves RJ, Shepheard SL. Pathophysiology of migraine - new insights. Can J Neurol Sci 1999;26(Suppl 2):S12-S19.

7. Product Monograph - Imitrex®, Glaxo Wellcome, 1999.

8. Product Monograph - Amerge ${ }^{\circledR}$, Glaxo Wellcome, 1998.

9. Product Monograph - Zomig®, Astra Zeneca, 1998.

10. Product Monograph - Maxalt ${ }^{\mathrm{TM}}$, Merck Frosst, 1999.

11. Meloche J. Triptans and migraine: which drug for which patient? Can J Diagn 1999; Jan:67-77.

12. Dixon R, Warrander A. The clinical pharmacokinetics of zolmitriptan. Cephalalgia 1997;17(Suppl 18):15-20.

13. Goldberg MR, Lowry RC, Musson DG, et al. Lack of pharmacokinetic and pharmacodynamic interaction between rizatriptan and paroxetine. J Clin Pharmacol 1999;39:192-199.

14. Rolan P. Potential drug interactions with the novel antimigraine compound zolmitriptan (Zomig $\left.{ }^{\mathrm{TM}}, 311 \mathrm{C} 90\right)$. Cephalalgia 1997;17 Suppl 18:21-27.

15. Mathew NT, Tietjen GE, Lucker C. Serotonin syndrome complicating migraine pharmacotherapy. Cephalalgia 1996;16:323-327.

16. Gardner DM, Lynd LD. Sumatriptan contraindications and the serotonin syndrome. Ann Pharmacother 1998;32:33-38.

17. Smith DA, Cleary EW, Watkins S, et al. Zolmitriptan (311C90) does not interact with fluoxetine in healthy volunteers. Int $\mathrm{J}$ Clin Pharmacol Ther 1998;36:301-305.
18. Peck RW, Seaber EJ, Dixon R, et al. The interaction between propranolol and the novel antimigraine agent zolmitriptan (311C90). Br J Clin Pharmacol 1997;44:595-599.

19. Scott AK, Walley T, Breckenridge AM, et al. Lack of an interaction between propranolol and sumatriptan. $\mathrm{Br} \mathrm{J}$ Clin Pharmacol 1991;32:581.

20. Gallagher RM, Dennish G, Spierings ELH, Chitra R. A comparative trial of zolmitriptan and sumatriptan for the acute oral treatment of migraine. Headache 2000;40:119-128.

21. Bomhof M, Paz J, Legg N, Allen C, Vandormael K, Patel K, and the Rizatriptan-Naratriptan Study Group. Comparison of rizatriptan $10 \mathrm{mg}$ vs. naratriptan $2.5 \mathrm{mg}$ in migraine. Eur Neurol 1999;42:173-179.

22. Visser WH, Terwindt GM, Reines SA, et al, for the Dutch/US Rizatriptan Study Group. Rizatriptan vs. sumatriptan in the acute treatment of migraine. Arch Neurol 1996;53:1132-1137.

23. Goldstein J, Ryan R, Jiang K, et al, and the Rizatriptan Protocol 046 Study Group. Crossover comparison of rizatriptan $5 \mathrm{mg}$ and 10 $\mathrm{mg}$ versus sumatriptan $25 \mathrm{mg}$ and $50 \mathrm{mg}$ in migraine. Headache 1998;38:737-747.

24. Tfelt-Hansen P, Teall J, Rodriguez $F$, et al, on behalf of the Rizatriptan 030 Study Group. Oral rizatriptan versus oral sumatriptan: a direct comparative study in the acute treatment of migraine. Headache 1998;38:748-755.

25. Silberstein SD. Migraine symptoms: results of a survey of selfreported migraineurs. Headache 1995;35:387-396.

26. Ferrari MD, James MH, Bates D, et al. Oral sumatriptan: effect of a second dose, and incidence and treatment of headache recurrences. Cephalalgia 1994;14:330-338.

27. Diamond S, Elkind A, Jackson RT, et al. Multiple-attack efficacy and tolerability of sumatriptan nasal spray in the treatment of migraine. Arch Fam Med 1998;7:234-240.

28. Cady RK, Dexter J, Sargent JD, et al. Efficacy of subcutaneous sumatriptan in repeated episodes of migraine. Neurology 1993;43:1363-1368

29. Bomhof MA, Heywood J, Pradalier A, et al. Tolerability and efficacy of naratriptan tablets with long-term treatment (6 months). Naratriptan Long-Term Study Group. Cephalalgia 1998; 18:33-37.

30. The International 311C90 Long-Term Study Group. The long-term tolerability and efficacy of oral zolmitriptan (Zomig, 311C90) in the acute treatment of migraine. An international study. Headache 1998:38:173-183.

31. Zagami AS, for the International 311C90 Long-Term Study Group. 311C90: long-term efficacy and tolerability profile for the acute treatment of migraine. Neurology 1997;48(Suppl 3):S25-S28.

32. Tepper SJ, Donnan GA, Dowson AJ, et al. A long-term study to maximize migraine relief with zolmitriptan. Curr Med Res Opin 1999;15:254-271.

33. Tuchman M, Edvinsson L, Geraud G, et al. Zolmitriptan provides consistent migraine relief when used in the long term. Curr Med Res Opin 1999;15:272-281.

34. Pfaffenrath V, Cunind G, Sjonell, et al. Efficacy and safety of sumatriptan tablets $(25 \mathrm{mg}, 50 \mathrm{mg}$, and $100 \mathrm{mg})$ in the acute treatment of migraine: defining the optimum doses of oral sumatriptan. Headache 1998;38:184-190.

35. Kramer MS, Matzura-Wolfe D, Polis A, et al. A placebo-controlled crossover study of rizatriptan in the treatment of multiple migraine attacks. Neurology 1998;51:773-781.

36. Mathew NT, Asgharnejad M, Peykamian M, Laurenza A, on behalf of the Naratriptan S2WA3003 Study Group. Naratriptan is effective and well-tolerated in the acute treatment of migraine. Results of a double-blind, placebo-controlled, crossover study. Neurology 1997;49:1485-1490.

37. Klassen A, Elkind A, Asgharnejad M, et al, on behalf of the Naratriptan S2WA3001 Study Group. Naratriptan is effective and well-tolerated in the acute treatment of migraine. Results of a double-blind, placebo-controlled, parallel-group study. Headache 1997;37:640-645.

38. O'Quinn S, Davis RL, Gutterman DL, et al. Prospective large-scale study of the tolerability of subcutaneous sumatriptan injection for acute treatment of migraine. Cephalalgia 1999;19:223-231. 
39. Maassen VanDenBrink AM, Reekers M, Bax WA, et al. Coronary side-effect potential of current and prospective antimigraine drugs. Circulation 1998;98:25-30.

40. Ephross SA. Sumatriptan and Naratriptan Pregnancy Registries Advisory Committee, Glaxo Wellcome, U.S.A. Interim results from the sumatriptan pregnancy registry [abstract]. Headache 1999;39:354

41. Oleson C, Steffensen FH, Sorensen HT, et al. Pregnancy outcome following prescription for sumatriptan. Headache 2000;40:20-24.

42. Briggs GG, Freeman RK, Yaffe SJ. Drugs in pregnancy and lactation. 5th ed. Baltimore (MD): Williams \& Wilkins; 1998.

43. Teall J, Tuchman M, Cutler N, et al. Rizatriptan (MAXALT) for the acute treatment of migraine and migraine recurrence. A placebocontrolled, outpatient study. Headache 1998;38:281-287.

44. Lipton RB, Stewart WF. Clinical applications of zolmitriptan (Zomig, 311C90). Cephalalgia 1997; 17:53-59.

45. Solomon GD, Cady RK, Klapper JA, et al. Clinical efficacy and tolerability of $2.5 \mathrm{mg}$ zolmitriptan for the acute treatment of migraine. Neurology 1997;49:1219-1225.

46. Sheftell F, O'Quinn S, Watson C, et al. Low migraine headache recurrence with naratriptan: clinical parameters related to recurrence. Headache 2000;40:103-110.

47. Mathew NT, Kailasam F, Meadors L, et al. Treatment of oral sumatriptan nonresponders with $5 \mathrm{mg}$ zolmitriptan and $10 \mathrm{mg}$ rizatriptan: a comparative open trial [abstract]. Headache 1999;39:368.

48. Limmroth V, Kazarawa Z, Fritsche G, Diener H-C. Headache after frequent use of serotonin agonists zolmitriptan and naratriptan [research letter]. Lancet 1999;353:378.

49. Evers S, Gralow I, Bauer B, et al. Sumatriptan and ergotamine overuse and drug-induced headache: a clinicoepidemiologic study. Clin Neuropharmacol 1999;22:201-206.

50. Gaist D, Tsiropoulos I, Sindrup SH, et al. Inappropriate use of sumatriptan: population based register and interview study. BMJ 1998;316:1352-1353.

51. The Sumatriptan Auto-Injector Study Group. Self-treatment of acute migraine with subcutaneous sumatriptan using an auto-injector device. Eur Neurol 1991;31:323-331.

52. Gross MLP, Kay J, Turner AM, et al. Sumatriptan in acute migraine using a novel cartridge system self-injector. Headache 1994;34:559-563.

53. Mathew NT, Dexter J, Couch J, et al. Dose ranging efficacy and safety of subcutaneous sumatriptan in the acute treatment of migraine. Arch Neurol 1992;49:1271-1276.

54. Russell MB, Holm-Thomsen OE, Rishoj Nielson M, et al. A randomized double-blind placebo-controlled crossover study of subcutaneous sumatriptan in general practice. Cephalalgia 1994; 14:291-296.

55. Mushet GR, Cady RK, Baker CC, et al. Efficacy and tolerability of subcutaneous sumatriptan administered using the IMITREX STATdose system. Clin Therap 1996;18:687-699.

56. Jensen K, Tfelt-Hansen P, Hansen EW, et al. Introduction of a novel self-injector for sumatriptan. A controlled clinical trial in general practice. Cephalalgia 1995;15:423-429.
57. Diamond S, Elkind A, Jackson RT, et al. Multiple-attack efficacy and tolerability of sumatriptan nasal spray in the treatment of migraine. Arch Fam Med 1998;7:234-240.

58. Gijsman H, Kramer MS, Sargent J, et al. Double-blind, placebocontrolled, dose-finding study of rizatriptan (MK-462) in the acute treatment of migraine. Cephalalgia 1997; 17:647-651.

59. Ahrens SP, Farmer MV, Williams DL, et al. Efficacy and safety of rizatriptan wafer for the acute treatment of migraine. Cephalalgia 1999; 19:525-530.

60. Rapoport AM, Ramadan NM, Adelman JU, et al. Optimizing the dose of zolmitriptan (Zomig,*311C90) for the acute treatment of migraine. A multicenter, double-blind, placebo-controlled, dose range-finding study. Neurology 1997; 49:1210-1218.

61. Solomon GD, Cady RK, Klapper JA, et al. Clinical efficacy and tolerability of $2.5 \mathrm{mg}$ zolmitriptan for the acute treatment of migraine. Neurology 1997; 49:1219-1225.

62. The Oral Sumatriptan Dose-Defining Study Group. Sumatriptan an oral dose-defining study. Eur Neurol 1991; 31:300-305.

63. The Oral Sumatriptan International Multiple-Dose Study Group. Evaluation of a multiple-dose regimen of oral sumatriptan for the acute treatment of migraine. Eur Neurol 1991; 31:306-313.

64. Goadsby PJ, Zagami AS, Donnan GA, et al. Oral sumatriptan in acute migraine. Lancet 1991; 338:782-783.

65. Cutler N, Mushet GR, Davis R, et al. Oral sumatriptan for the acute treatment of migraine: evaluation of three dosage strengths. Neurology 1995; 45(Suppl 7):S5-S9.

66. Sargent J, Kirchner JR, Davis R, et al. Oral sumatriptan is effective and well tolerated for the acute treatment of migraine: results of a multicenter study. Neurology 1995; 45(Suppl 7):S10-S14.

67. Nappi G, Sicuteri F, Byrne M, et al. Oral sumatriptan compared with placebo in the acute treatment of migraine. J Neurol 1994; 241:138-144.

68. Centonze V, Polito MB, Di Bari M, et al. Valutazione dell'efficacia di sumatriptan nella terapia orale dell'attacco di emicrania. Resultati clinici. Clin Ter 1995; 146:721-728.

69. Patten JP, for the Oral Sumatriptan Dose-Defining Study Group. Clinical experience with oral sumatriptan: a placebo-controlled, dose-ranging study. J Neurol 1991; 238:S62-S65.

70. Tfelt-Hansen P, Henry P, Mulder LJ, et al. The effectiveness of combined oral lysine acetylsalicylate and metoclopramide compared with oral sumatriptan for migraine. Lancet 1995; 346:923-926.

71. Rederich G, Rapoport A, Cutler N, et al. Oral sumatriptan for the long-term treatment of migraine: clinical findings. Neurology 1995; 45(Suppl 7):S15-S20.

72. Dahlof C, Hogenhuis L, Oleson J, et al. Early experience with subcutaneous naratriptan in the acute treatment of migraine: a dose-ranging study. Eur J Neurol 1998; 5:469-477.

73. Salonen R, Ashford E, Dahlof C, et al. Intranasal sumatriptan for the acute treatment of migraine. International Intranasal Sumatriptan Study Group. J Neurol 1994; 241:463-469.

74. Ryan R, Elkind A, Baker CC, et al. Sumatriptan nasal spray for the acute treatment of migraine. Results of two clinical studies. Neurology 1997; 49:1225-1230. 